\title{
Efficacy of Cetuximab in Combination with FOLFIRI in a Patient with KRAS Wild-Type Metastatic Anal Cancer
}

\author{
Hanna Barmettler ${ }^{a} \quad$ Paul Komminoth $^{b} \quad$ Mathias Schmid ${ }^{a}$ \\ Donat Duerra \\ ${ }^{a}$ Clinic of Medical Oncology and Hematology and ${ }^{b}$ Department of Pathology, \\ City Hospital Triemli, Zurich, Switzerland
}

\section{Key Words}

Metastatic anal cancer $\cdot$ Cetuximab - FOLFIRI - KRAS - EGFR

\begin{abstract}
Anal canal cancer is a rare tumor without clear treatment evidence in the metastatic setting. In terms of the bad prognosis of patients with metastatic anal cancer, further therapeutic options are urgently needed. In this paper we present the case of a 64-year-old man suffering from undifferentiated squamous cell carcinoma with liver metastases. After progression on cisplatin and fluorouracil, tumor tissue was analyzed with respect to antiEGFR therapy with cetuximab. There was no KRAS mutation and the EGFR expression level in the tumor tissue was 2+; ideal conditions for the immunotherapy. Encouraged by these results we started a therapy using FOLFIRI in combination with cetuximab. Fortunately the patient showed a partial response after 6 cycles. On patient's preference we did a therapy break of 6 weeks. Within this time period the disease was progressive indicating its aggressiveness. However, the same immunotherapy was able to stabilize the disease for a further 3 months. The patient died 21 months after diagnosis because of liver failure. Nevertheless, from our perspective the combination of FOLFIRI and cetuximab is quite a promising therapeutic option for patients with metastatic anal cancer. Potential predictive factors of the immunochemotherapy are discussed in this paper.
\end{abstract}

\section{Background}

Anal cancers represent rare gastrointestinal tumors with an estimated annual incidence of about 5,820 new cases in the United States in 2011 [1]. Fortunately, only $10-20 \%$ of patients present extrapelvic metastases at the beginning or during their 
illness [2]. The most frequently seen metastatic sites are liver, lung, and extrapelvic lymph nodes. Only few data about chemotherapy protocols have been published for metastatic anal carcinoma. There is some evidence that fluorouracil (5-FU)-based chemotherapy plus cisplatin has some benefit with a response rate of about $50 \%$ and a median overall survival of 13-55 months [3-5]. In selected patients, an aggressive multimodality approach including resection of metastases, irradiation of para-aortic lymph nodes or intra-arterial chemotherapy results in long-term survival [6, 7]. In this article, we report about a 64-year-old male patient with metastatic anal cancer who failed first-line palliative therapy with cisplatin and 5-FU but who showed a good response to second-line therapy with irinotecan, leucovorin, 5-FU and cetuximab, a monoclonal antibody against the epidermal growth factor receptor (EGFR).

\section{Case Presentation}

A 64-year-old previously healthy man underwent routine colonoscopy. An incidental tumor in the anal canal was detected in this HIV-negative, heterosexual patient. The histological examination of the anal lesion showed an undifferentiated squamous cell carcinoma. Two lesions in the right lobe of the liver were detected by CT scan (fig. 1). The histological result of the biopsy of these liver lesions was identical with the primary tumor. Considering the fact that the primary anal lesion was very small (cT1) and the patient was in an excellent physical shape (ECOG 0), we discussed at our interdisciplinary tumor board a potentially curative approach with a local excision of the small tumor in the anal canal followed by neoadjuvant chemotherapy with 5 -FU and cisplatin and subsequent resection of the liver metastases. Six weeks after the R0 transanal tumor resection without rectal amputation, chemotherapy containing cisplatin $\left(70 \mathrm{mg} / \mathrm{m}^{2}\right.$ on days 1 and 2$)$ and 5 -FU $\left(1,000 \mathrm{mg} / \mathrm{m}^{2}\right.$ on days 1-4, repeated every 4 weeks) was started. After 3 cycles, CT scan showed progressive liver disease. In contrast to the previous study there were now metastases on both lobes of the liver, making a curative liver resection quite challenging. However, there was no evidence of a relapse at the primary tumor side. Based on the report of Lukan et al. [8], who published quite promising results about metastatic anal cancer patients treated with cetuximab, a monoclonal antibody against EGFR, in combination with irinotecan, we excluded a KRAS mutation by DNA sequencing in the tumor material of our patient. Second-line therapy with cetuximab $\left(500 \mathrm{mg} / \mathrm{m}^{2}\right.$ every other week after a loading dose of $400 \mathrm{mg} / \mathrm{m}^{2}$ one week before) in combination with irinotecan $\left(180 \mathrm{mg} / \mathrm{m}^{2}\right)$, leucovorin $(200$ $\left.\mathrm{mg} / \mathrm{m}^{2}\right)$ and 5 -FU $\left(400 \mathrm{mg} / \mathrm{m}^{2}\right)$ as bolus injection followed by a continuous infusion $\left(2.4 \mathrm{~g} / \mathrm{m}^{2}\right)$ during $48 \mathrm{~h}$ every 2 weeks (FOLFIRI) was initiated. After 6 cycles, the CT scan showed a partial remission of the liver lesions according the RECIST 1.1 criteria (fig. 2). Hereupon, the patient asked for a therapy break. Six weeks later, the CT scan was repeated and showed a clear progression of the known liver lesions as well as several new liver lesions. The same treatment protocol was restarted. Fortunately, the patient tolerated the therapy extremely well and did not suffer from any severe side effects. After additional 7 cycles, a mixed response based on CT scan could be demonstrated. Given the patient's wish for a new treatment break for spending four weeks in an Ayurveda Clinic in India we stopped the therapy again. Two months later, the images again showed already a progressive disease with an increase in the size of the known liver metastases. Selective internal radiation therapy (SIRT) for treating the liver metastases was evaluated; however, this was not an option in his case because of a distinctive shunt between the liver and the lung. In the meantime the patient was already in a reduced physical shape (ECOG score 1-2). Nevertheless, he wanted to be treated and his liver values were still in the normal range so that we offered him a reduced therapy regimen containing irinotecan and cetuximab, but no leucovorin and 5-FU. The dosages of cetuximab and irinotecan were unchanged; the treatment interval was stretched to 3 weeks on the patient's preference. Unfortunately the patient did worse quite soon and therefore treatment was stopped after 8 weeks. He died because of liver failure a few weeks after therapy cessation and 21 months after diagnosis. 


\section{Conclusion}

Anal cancer is an uncommon tumor. It compromises only about $2 \%$ of all gastrointestinal malignancies. $10-20 \%$ of all these patients develop metastatic disease. Based on the low incidence and the low metastatic risk very limited data about treatment options for patients with metastatic anal cancer are available. These few data indicate that 5-FU-based chemotherapy in combination with cisplatin may have some benefit [3-5]. However, this observation has not yet been proven by a statistically powered, prospectively conducted, placebo-controlled study. Nevertheless, most physicians consider the combination of cisplatin and 5-FU as a standard of care for their patients with metastatic anal cancer. After failure of this therapy, the literature is quite disappointing with respect to effective chemotherapy protocols. New therapeutic options are urgently mandatory.

EGFR is expressed by multiple epithelial tumors. Blocking of this receptor by a monoclonal antibody such as cetuximab is of clinical benefit in the metastatic setting of several tumors such as head and neck [9], lung [10] and colorectal cancer [11]. Anal canal cancer tissue expresses nearly universal EGFR [12-14], as also demonstrated in our patient. Based on these findings, cetuximab might be an ideal candidate for the improvement of the therapy in anal cancer patients. However, not all of the anal cancer patients described in the literature showed a response to cetuximab [8]. In a retrospective analysis of anal cancer patients treated with this monoclonal antibody in combination with irinotecan, Lukan et al. [8] could show partial remission or at least tumor stabilization in KRAS wild-type patients, whereas patients harboring KRAS mutations had progressive disease. In analogy to colorectal patients one could speculate that KRAS might be a predictive factor for cetuximab therapy in metastatic anal cancer patients. However, this hypothesis has to be confirmed by a prospectively designed study. In the case of our patient the administration of cetuximab depended on the result of KRAS mutation status, which is in accordance with actual retrospective data [8]. Nevertheless, our case report further supports the necessity of a prospective, randomized, placebo-controlled study. The fact that the majority of anal cancers harbor no KRAS mutation $[12,13]$ makes cetuximab an interesting option for the treatment of anal canal patients in order to improve their clinical outcome. However, it might be absolutely possible that there are further predictive factors with respect to the cetuximab therapy in anal cancer patients. A recently published study showed that cetuximab in lung cancer was just beneficial in patients with EGFR tumor expression level above a specific threshold [15] and EGFR gene amplification might therefore be another potential predictive factor. Besides cetuximab, the chemotherapeutic partners might also be important for the response rate of the immunotherapy. Our patient was treated with cetuximab in combination with FOLFIRI (scheme see above). This combination has been extensively described in colorectal but not in anal cancer patients. To our knowledge, this is the first report in the literature about this combination in metastatic anal cancer patients. In contrast to other published combinations of cetuximab, irinotecan, 5-FU and leucovorin as 5-FU modulator were added. 5-FU plays a major role in the treatment of anal cancer. This statement is supported by the fact that 5 -FU is incorporated in adjuvant as well as in palliative standard chemotherapy protocols for anal cancer patients. Therefore we believe that adding 5-FU in combination with leucovorin increases the efficiency of our therapy. In addition, we know that multi-agent chemotherapy has in general a higher risk of 
toxicity than single-agent chemotherapy. The higher toxicity might overcome the benefit of the multi-agent therapy. Fortunately our patient did not suffer from severe side effects despite our multi-agent approach. As single grade II toxicity, he developed a skin rush about two weeks after the start of cetuximab as a typical side effect of this antibody. It disappeared quite soon after starting topical therapies with lotions. Other toxicities were not observed. The combination of cetuximab with FOLFIRI was extensively analyzed in colorectal patients. There it was extremely well tolerated [11]. It would be very surprising if there would be a big difference with respect to toxicity in these two patient populations. Therefore we believe that FOLFIRI might be an effective chemotherapeutic regimen in combination with cetuximab; probably even more effective than irinotecan alone.

In summary, the combination of cetuximab with FOLFIRI showed a promising response in our KRAS wild-type anal cancer patient. However, further investigations are urgently needed.

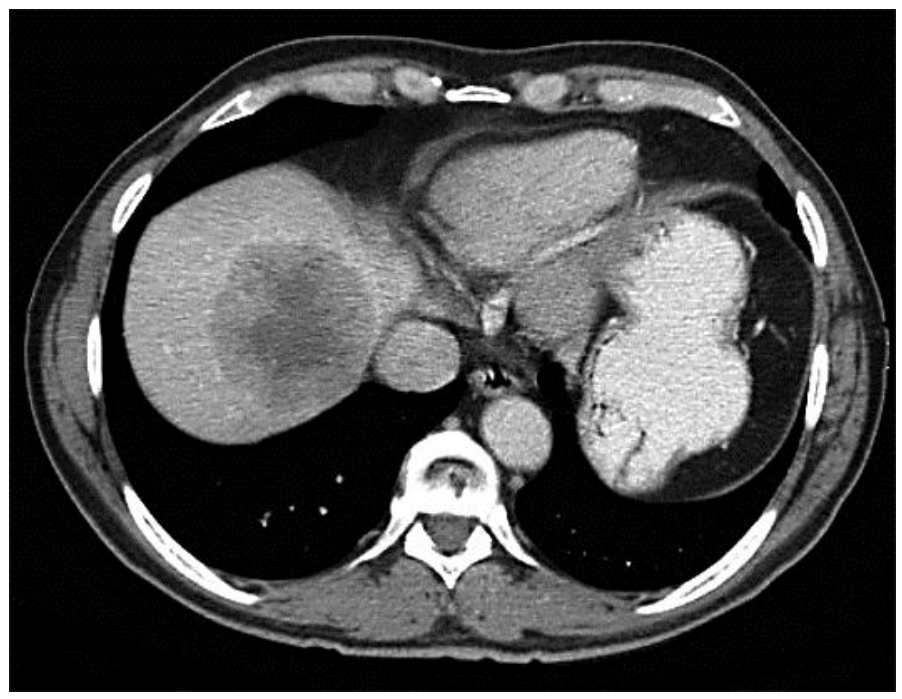

Fig. 1. Before chemoimmunotherapy with FOLFIRI and cetuximab. 


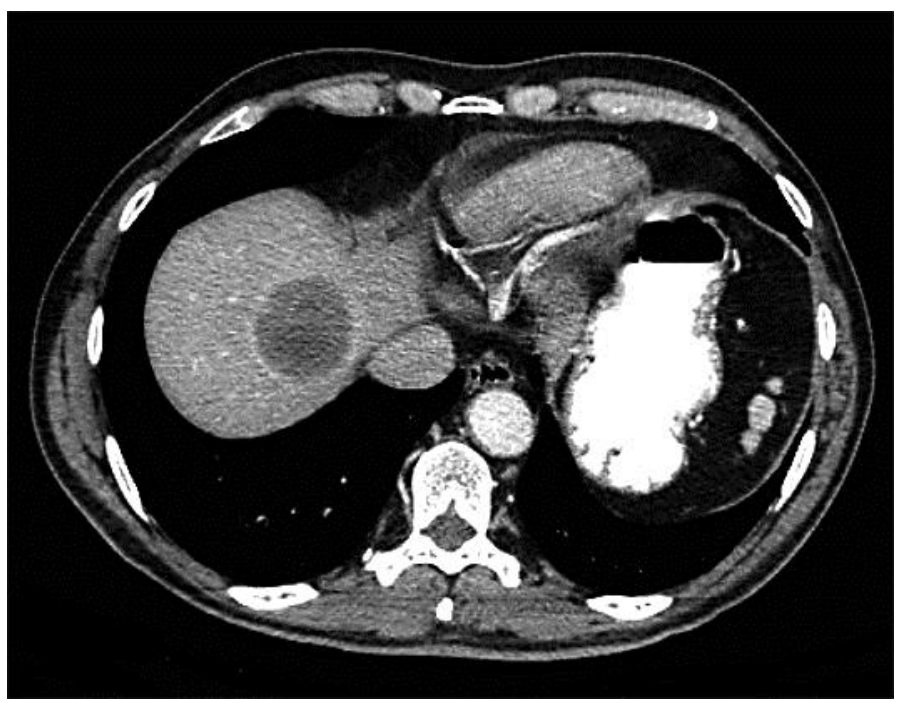

Fig. 2. After 6 cycles of chemoimmunotherapy with FOLFIRI and cetuximab.

\section{References}

-1 Siegel R, Ward E, Brawely 0, Jemal A: Cancer statistics, 2011: The impact of eliminating socioeconomics and racial disparities on premature cancer deaths. CA Cancer J Clin 2011;61:212-236.

2 Cummings BJ: Metastatic anal cancer: the search for cure. Onkologie 2006;29:5-6.

-3 Jaiyesimi IA, Pazdur R: Cisplatin and 5-fluorouracil as salvage therapy for recurrent metastatic squamous cell carcinoma of the anal canal. Am J Clin Oncol 1993;16:536-540.

-4 Ajani JA, Carrasco CH, Jackson DE, Wallace S: Combination of cisplatin plus fluoropyrimidine chemotherapy effective against liver metastases from carcinoma of the anal canal. Am J Med 1989;87:221-224.

5 Faivre C, Rougier P, Ducreux M, Mitry E, Lusinchi A, Lasser P, Elias D, Eschwege F: [5-fluorouracil and cisplatin combination chemotherapy for metastatic squamous-cell anal cancer] Carcinome épidermoïde métastatique de l'anus: étude retrospective de l'efficacité de l'association de 5-fluorouracil en perfusion continue et de cisplatine. Bull Canc 1999;86:861-865.

-6 Wanebo HJ, Belliveau J, Begossi G, Levy A: Isolated chemotherapeutic perfusion of the pelvis for advanced rectal cancer. Colorectal Dis 2003;5:508-514.

7 Tokar M, Bobilev D, Zalmanov S, Geffen DB, Walfisch S: Combined multimodality approach to the treatment of metastatic anal carcinoma: report of a case and review of the literature. Onkologie 2006;29:5-6.

8 Lukan N, Stroebel P, Willer A, Kripp M, Dinter D, Mai S, Hochhaus A, Hofheinz RD: Cetuximab-based treatment of metastatic anal cancer: correlation of response with KRAS mutational status. Oncology 2009;77:293-299.

-9 Burtness B, Goldwasser MA, Flood W, Mattar B, Forastiere AA: Phase III randomized trial of cisplatin plus placebo compared with cisplatin and cetuximab in metastatic/recurrent head and neck cancer: an Eastern Cooperative Oncology Group study. J Clin Oncol 2005;23:8646-8654.

10 Pirker R, Pereira JR, Szczesna A, von Pawel J, Krzakowski M, Lamlau R, Vynnychenko I, Park K, Yu CT, Ganul V, Roh JK, Bajetta E, O’Byrne K, de Marinis F, Eberhardt W, Goddemeier T, Emig M, Gatzemeir U: Cetuximab plus chemotherapy in patients with advanced non-small-cell lung cancer (FLEX): an openlabel randomised phase III trial. Lancet 2009;373:1525-1531.

11 Van Cutsem E, Köhne CH, Hitre E, Zaluski J, Chang Chien CR, Makhson A, D’Haens G, Pintér T, Lim R, Bodoky G, Roh JK, Folprecht G, Ruff P, Stroh C, Tejpar S, Schlichting M, Nippgen J, Rougier P: Cetuximab and chemotherapy as initial treatment for metastatic colorectal cancer. N Engl J Med 2009;360:14081417. 
12 Van Damme N, Deron P, Van Roy N, Demetter P, Bols A, Van Dorpe J, Baert F, Van Laethem JL, Speleman F, Pauwels P, Peeters M: Epidermal growth factor receptor and K-RAS status in two cohorts of squamous cell carcinomas. BMC Cancer 2010;10:189-197.

13 Zampino MG, Magni E, Sonzogni A, Renne G: K-ras status in squamous cell anal carcinoma (SCC): it's time for target-oriented treatment? Cancer Chemother Pharmacol 2009;65:197-199.

14 Van Damme N, van Roy N, Speleman F, Bols A, van Dorpe J, Pauwels P, Peeters M: EGFR and KRAS gene status evaluation in anal canal squamous cell carcinoma. J Clin Oncol 2008;26(suppl):15569.

-15 Celik I, O’Byrne KJ: EGFR expression as a predictor of survival for first-line chemotherapy plus cetuximab in patients with advanced non-small-cell lung cancer: analysis of data from phase 3 FLEX study. Lancet Oncol 2012;13:33-42. 